\title{
Table of a Weierstrass Continuous Non-Differentiable Function
}

\author{
By Herbert E. Salzer and Norman Levine
}

Many studies have been made of continuous non-differentiahle functions [1], the most famous of which is Weierstrass's $W(a, b, x)$ defined by

$$
W(a, b, x)=\sum_{n=1}^{\infty} a^{n} \cos \left(b^{n} \pi x\right), \quad 0<a<1, b \text { an odd integer. }
$$

It is shown in some books [1], [2] that for

$$
a b>1+\frac{3 \pi}{2}
$$

$\mathrm{II}(a, b, x)$ is continuous everywhere and has no derivative anywhere, but Bromwich [3] improved this condition to

$$
\text { al) }>1+\frac{3 \pi}{2}(1-a),
$$

which, according to Hardy [4] is the sharpest result (as of 1916) for no derivative, finite or infinite. (Hardy showed $b>1, a b \geqq 1$ sufficient to establish the non-existence of any finite derivative. He also showed that those same conditions, together with $a(b+1)<2$ for $b=4 k+1$, permitted the existence of an infinite derivative at certain points.) To illustrate the difference between (2) and (3) for $a=\frac{1}{2}$, (2) requires $b \geqq 13$, while (3) permits $b=7$. However, as far as the authors know there may be considerable work to be done in the direction of lowering the bound of $1+\frac{3 \pi}{2}(1-a)$ in $(3)$ for the case of no derivative, finite or infinite.

Owing to the unusual nature of $W(a, b, x)$ and the absence of any previous table, or even graph, despite the countless number of theoretical papers, it was believed that an extensive table of this Weierstrass function for some typical pair of parameters $a$ and $b$ might be of value as more than a mere curiosity, namely for suggesting or motivating further research, and for its interest to workers in numerical analysis. Thus, in this last connection, it might be of interest to determine empirically what results in numerical integration and possibly interpolation are available from the continuity alone. That $W(a, b, x)$ is integrable follows from its continuity, and one might be curious to see the results of applying standard numerical integration formulas where the usual derivative formulas for the remainder would the inapplical,le. Likewise, one might be curious to test out standard Lagrangian interpolation, where the remainder is often expressed in terms of derivatives. (We can write down interpolation and numerical integration formulas, avoiding derivatives in the remainder terms by employing divided differences and integrals with divided differences in the integrand, respectively. However, one usually estimates divided differences in terms of derivatives.) Finally, one's curiosity might extend as far as

Received February 23, 1960; revised July 28, 1960. 
glancing at the results of standard numerical differentiation and interpretation of the results in the light of the knowledge that $W(a, b, x)$ has no derivative.

For tabulation of any $W(a, b, x)$, it is immediately apparent from (1) that

$$
W(a, b, 1+x)=-W(a, b, x),
$$

so that the range of $x$ need not go outside $(0,1)$. From (1),

$$
\begin{aligned}
& W(a, b, 0)=-W(a, b, 1)=a /(1-a) ; \\
& W\left(a, b, \frac{1}{2}\right)=0 .
\end{aligned}
$$

From the trigonometric identity

$$
\cos \left(m \pi\left(\frac{1}{2} \pm t\right)\right)=\mp(-1)^{(m-1) / 2} \sin m \pi t, m \text { odd },
$$

we have

$$
W\left(a, b, \frac{1}{2}+t\right)=-W\left(a, b, \frac{1}{2}-t\right),
$$

so that for complete tabulation of any $W(a, b, x)$ it suffices for $x$ to range from 0 to $\frac{1}{3}$.

In connection with the choice of $a$ and $b$, it is apparent that for $a$ close to 1 , we can choose $b$ as low as 3 , but the convergence of the series in (1) would be ton slow for practical calculation of $W(a, b, x)$ to high accuracy. Making $a$ very small would give rapid convergence, but for accuracy fixed at a certain number of decimal places as $a$ tends to get very small, say

$$
a=\epsilon, \quad b^{n}>N=\left\{1+\frac{3 \pi}{2}(1-\epsilon)\right\}^{n} / \epsilon^{n}
$$

becomes enormous and $W(\epsilon, b, x)$ becomes essentially the first term of $(1), \epsilon \cos$ $\left(b^{n} \pi x\right)$, whose graph would appear like that of a very highly oscillatory function of small amplitude. As a compromise between these two extreme types, we took $a=\frac{1}{2}$ and $b=7$. The choice $a=\frac{1}{2}$ did not lead to too many terms of (1), 50 terms giving a truncating error $<\frac{1}{2} \cdot 10^{-15}$, and yet there were sufficient terms beyond the first few to give a graph that is characteristic of $W(a, b, x)$ rather than a predominantly sinusoidal type of curve. The $b=7$ barely satisfies (3), thus tending to minimize the oscillatory behavior of $W(a, b, x)$ and to facilitate graphing. We shall denote $W(a, b, x)$ which is tabulated here for $a=\frac{1}{2}$ and $b=7$ by $W(x)$.

This present table of $W(x), x=0(.001) 1$ to $12 \mathrm{D}$; was printed out and rounded from a preliminary calculation on the IBM 704 to several more places. Two separate and independent print-outs, supposedly identical, were proofread against each other, with just a single print-out error turning up. Naturally, no differencing check could be made upon the correctness of this table of $W(x)$, but every value underwent the following final functional check:

$$
W(7 x)=2 W(x)-\cos (7 \pi x),
$$

which was performed by desk calculation upon $W(x)$ on one of the preliminary print-outs. The results showed $W(x)$ to be correct to around 14D. In employing (8), $W(7 x)$ was found in the table as $\pm W\left(x^{\prime}\right)$ for some suitable $x^{\prime}, 0 \leqq x^{\prime} \leqq \frac{1}{2}$, according to (4) and (7), and $\cos (7 \pi x)$, after reduction of $7 \pi x$ to the first quadrant, was 

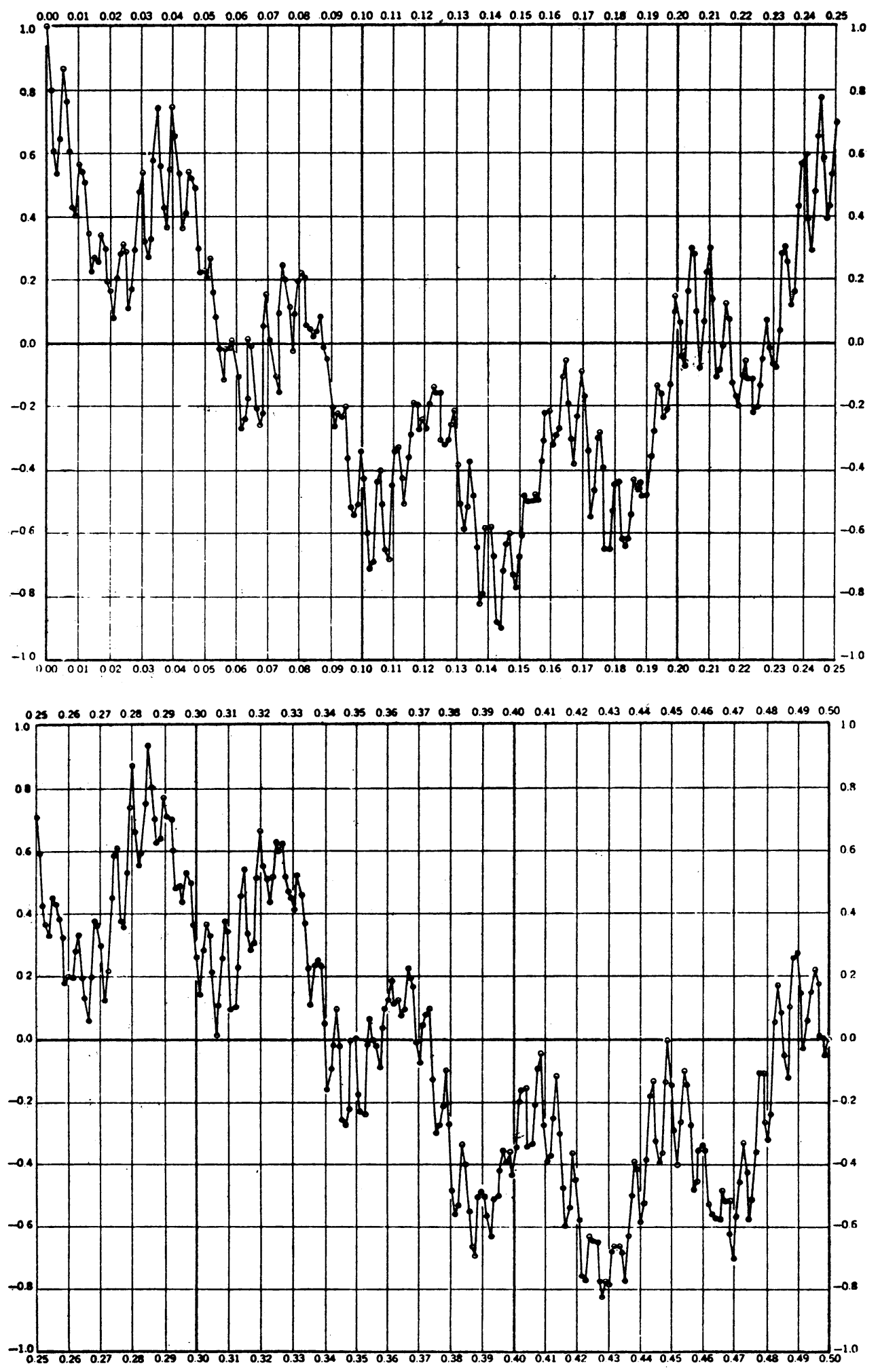

Fig. 1.-Fllustration of a Weierstrass, Everywhere-Continuous Nowhere-Differentiable Function, $W(x)=\sum_{n=1}^{\infty} a^{n} \cos \left(b^{n} \pi x\right) a=\frac{1}{2} ; b=7 ; x=0(0.001) 0.500$ 
looked up in a well-known 15-place table at intervals of $0.01^{\circ}$ [5]. The final 12-decimal table was checked by reading it several times against one of the print-outs, and it is believed to be correct to well within a unit in the 12th decimal.

The purpose of the accompanying figure, which is merely a broken line graph of the table of $W(x)$, is to furnish at a glance a view of the peculiar behavior of $W(x)$. Of course, the graphical picture would be more complete if the time and means were available for calculating $W(a, b, x)$ as a function of $a$ also, and for a sequence of permissible odd integral values of $b$ (according to (3)) to correspond to each $a$. Although no offhand justification could be found for drawing anything smoother than a broken line connecting these 500 points, one still finds its ripples of irregularity, superposed upon a broader pattern of smoothness, to be quite revealing as to the nature of $W(x)$ and how it might appear under repeated "magnification" (i.e., subtabulation).

To establish (8), replace $x$ by $7 x$, in $W(x)=\sum_{n=1}^{\infty} \cos \left(7^{n} \pi x\right) / 2^{n}$, to get

$W(7 x)=2 \sum_{n=1}^{\infty} \cos \left(7^{n+1} \pi x\right) / 2^{n+1}=2 \sum_{n^{\prime}=2}^{\infty} \cos \left(7^{n^{\prime}} \pi x\right) / 2^{n^{\prime}}=2 W(x)-\cos (7 \pi x)$.

By repeated application of $(8)$,

$$
\begin{aligned}
& W\left(7^{n} x\right)=2 W\left(7^{n-1} x\right)-\cos \left(7^{n} \pi x\right)=4 W\left(7^{n-2} x\right)-2 \cos \left(7^{n-1} \pi x\right)-\cos \left(7^{n} \pi x\right) \\
& =8 W\left(7^{n-3} x\right)-4 \cos \left(7^{n-2} \pi x\right)-\cdots \text { etc. until we reach } \\
& (9) \quad W\left(7^{n} x\right)=2^{n} W(x)-\sum_{r=0}^{n-1} 2^{r} \cos \left(7^{n-r} \pi x\right) .
\end{aligned}
$$

From (9), for $x=1 / 7^{n}, W(1)=-1=2^{n} W\left(1 / 7^{n}\right)-\sum_{r=0}^{n-1} 2^{r} \cos \left(\pi / /^{r}\right)$, from which

$$
W\left(1 / 7^{n}\right)=-1 / 2^{n-1}+\sum_{r=1}^{n-1} \cos \left(\pi / 7^{r}\right) / 2^{n-r}
$$

Letting $n \rightarrow \infty$ in $(10)$, we see at once that

$$
\lim _{n \rightarrow \infty}\left\{\sum_{r=1}^{n-1} 2^{r} \cos \left(\pi / 7^{r}\right)\right\} / 2^{n}=1 .
$$

To test the value of standard numerical integration formulas upon $W(x)$, whose integral is given by

\begin{tabular}{|c|c|c|c|c|c|}
\hline Interval & True Value & Trapezoidal Rule & Deviation & Simpson's Rule & Deviation \\
\hline $\begin{array}{l}0 \text { to } 0.1 \\
0.1 \text { to } 0.2 \\
0.2 \text { to } 0.3 \\
0.3 \text { to } 0.4 \\
0.4 \text { to } 0.5\end{array}$ & $\begin{array}{r}0.0189929 \\
-0.0414565 \\
0.0308462 \\
0.0033770 \\
-0.0329802\end{array}$ & $\begin{array}{r}0.0189876 \\
-0.0414380 \\
0.0308443 \\
0.0034254 \\
-0.0330067\end{array}$ & $\begin{array}{r}-0.0000053 \\
+0.0000185 \\
-0.0000019 \\
+0.0000484 \\
-0.0000265\end{array}$ & $\begin{array}{r}0.0190144 \\
-0.0414543 \\
0.0308514 \\
0.0034027 \\
-0.0328827\end{array}$ & $\begin{array}{r}+0.0000215 \\
+0.0000022 \\
+0.0000052 \\
+0.0000257 \\
+0.0000975\end{array}$ \\
\hline
\end{tabular}

$$
\int_{0}^{x} W(t) d t=\frac{1}{\pi} \sum_{n=1}^{\infty} \sin \left(7^{n} \pi x\right) / 14^{n}
$$

the values of $\int_{0}^{0.1} W(t) d t, \int_{0.1}^{0.2} W(t) d t, \cdots, \int_{0.4}^{0.5} W(t) d t$ were computed analytically from (12), and then were computed numerically by both trapezoidal and Simpson's rules at intervals of 0.001 , with the following results: 
The results show no recognizable advantage in Simpson's rule. In fact, the sum of the absolute values of the above deviations in the trapezoidal rule is around $10^{-4}$, while the sum of the absolute values of the Simpson deviations is around $1 \frac{1}{2} \cdot 10^{-1}$. This may indicate that no higher-point formula will improve over the trapezoidal formula.

Lagrangian polynomial interpolation at intervals of 0.002 was tried for the 2through 7-point cases, for a mid-interval (i.e., already tabulated) value of $W(x)$ at two different places, $x=0.007$ and $x=0.037$, where the true value to $5 \mathrm{D}$ is 0.60807 and 0.43362 respectively. At each place the error in almost all cases ranged from around 0.01 to 0.05 . More specifically, for $x=0.007$ the error fluctuated between 0.01 for every even-point interpolation and 0.014 to 0.049 for various odd-point interpolations, and for $x=0.037$ there were deviations of 0.032 and 0.055 for respeetive 2-point and 3-point interpolation and deviations ranging from 0.001 to 0.021 in the higher-point interpolation. On the basis of these two tests alone it would appear that one could not really count upon any systematic improvement beyond linear interpolation.

Finally, out of pure curiosity, 2- through 7-point Lagrangian differentiation, for the "first derivative," was tried out at the tabular interval of 0.001 , for $x=0.002$, and surprisingly enough, outside of the 2-point answer of -74 and the 3-point answer of -133 , the remaining four cases all came within 6 units of -150 .

From a casual look at the graph of $W(x)$, it is apparent that in place of the derivative there is a general directional trend from any point $x_{0}$ if we do not go too far a way from $x_{0}$, and we might seek a suitable quantitative estimate for an "average slope" between $x_{0}$ and $x_{0}+\cdot h$. (The discussion here is concerned with a suitable generalization of the left- or right-hand derivative, rather than the derivative.) One suggestion that would appear natural for $W(x, a, b)$, or any other continuous function, would be to investigate the possibilities of the average of the difference quotient $\left\{f(x)-f\left(x_{0}\right)\right\} /\left(x-x_{0}\right)$, which exists and is itself continuous for every $x$ except $x_{0}$ in the open interval $\left(x_{0}, x_{0}+h\right)$. This average difference quotient or $D_{h} f\left(x_{0}\right)$ might have the following definition (assuming that it exists in the first place):

$$
D_{h} f\left(x_{0}\right)=\frac{1}{h} \int_{x_{0}}^{x_{0}+h}\left\{\left[f(x)-f\left(x_{0}\right)\right] /\left(x-x_{0}\right)\right\} d x .
$$

That (13) may be a suitable generalization follows from the fact that when $f^{\prime}\left(x_{0}\right)$ exists, (13) exists, and

$$
\lim _{h \rightarrow 0} D_{h} f\left(x_{0}\right)=f^{\prime}\left(x_{0}\right) .
$$

This is seen at once from the replacement of $\left\{f(x)-f\left(x_{0}\right)\right\} /\left(x-x_{0}\right)$ by $f^{\prime}\left(x_{0}\right)+$ $\epsilon(x)$ in (13) and the continuity of $\epsilon(x)$ in the closed set $\left(x_{0}, x_{0}+h\right)$ which makes $\epsilon(x)$ integrable. Thus (13) exists and

$$
\left|\frac{1}{h} \int_{x_{0}}^{x_{0}+h} \cdot \epsilon(x) d x\right| \rightarrow 0 \text { as } h \rightarrow 0
$$

which implies (14).

It is not difficult to find examples of continuous functions $f(x)$ where $f^{\prime}\left(x_{0}\right)$ does not exist and (a) also $D_{h} f\left(x_{0}\right)$ does not exist, or (b) $D_{h} f\left(x_{0}\right)$ exists but $\lim _{h \rightarrow 0}$ $D_{h} f\left(x_{0}\right)$ does not exist. But we may also have (c) no $f^{\prime}\left(x_{0}\right)$, with both $D_{h} f\left(x_{0}\right)$ and $\lim _{h \rightarrow 0} D_{h} f\left(x_{0}\right)$ existing. In other words the existence of $\lim _{h \rightarrow 0} D_{h} f\left(x_{0}\right)$ still 
does not imply the existence of $f^{\prime}\left(x_{0}\right)$. Such a counter-example, ${ }^{*}$ which is due to the referee, is the following. Let $x_{0}=0$ and

$$
\begin{aligned}
& f(x)=x \sin \frac{1}{x} \\
& f(0)=0 .
\end{aligned}
$$

This continuous function has no derivative at $x=0$, but

$$
\lim _{h \rightarrow 0} D_{h} f(0)=0 \text {. }
$$

First

$$
D_{h}=\frac{1}{h} \int_{0}^{h} \sin \left(\frac{1}{x}\right) d x
$$

exists since the integrand is bounded and continuous except at one point. This suffices. To estimate $D_{k}$ we let

$$
I_{n}=\int_{1 /(n+1) \pi}^{1 / n \pi} \sin \left(\frac{1}{x}\right) d x=\int_{n \pi}^{(n+1) \pi} \frac{1}{y^{2}} \sin y d y .
$$

By the mean value theorem

$$
I_{n}=(-1)^{n} \cdot 2 / \theta_{n}^{2}
$$

where

$$
n_{\pi}<\theta_{n}<(n+1) \pi \text {. }
$$

Suppose that $h=1 /(n+a) \pi, 0 \leqq a<1$. Then

$$
D_{h}=(n+a) \pi\left[\int_{(n+a) \pi}^{(n+1) \pi} y^{-2} \sin y d y+I_{n+1}+I_{n+2}+\cdots\right] \text {, }
$$

and therefore $\left|D_{h}\right|<(n+a) \pi\left|I_{n}\right|<2(n+a) \pi / n^{2} \pi^{2}$.

Therefore as $h \rightarrow 0, D_{h}$ also $\rightarrow 0$.

The authors wish to acknowledge the assistance of Mrs. Charlene M. Janos in checking the entire table of $W(x)$ by the functional check (8).

Convair-Astronautics

San Diego, California

1. A. N. Singr, The Theory and Construction of Non-Differentiable Functions, Lucknow University Studies, Faculty of Science, no. 1, 1935, reprinted in Squaring the Circle and Other Monographs, Chelsea Publishing Co., New York, 1953.

2. E. Goursat, A Course in Mathematical Analysis, Vol. 1, translated by E. R. Hedrick, Ginn \& Co., Boston, 1904, p. 423-425.

3. T. Bromwich, An Introduction to the Theory of Infinite Series, Macmillan \& Co., Ltd., London, 1908, p. $490-491$. Note: The proof of the sufficiency of $a b>1+\frac{3 \pi}{2}(1-a)$ is not contained in the later 1926 edition.

4. G. H. HARDY, "Weierstrass's non-differentiable function," Trans. Amer. Math. Soc., v. 17, 1916, p. 301-325.

5. Nat. Bur. Standards Appl. Math.Ser. No. 5, Table of Sines and Cosines to Fifteen Decimal Places at Hundredths of a Degree, U. S. Government Printing Office, Washington 25, D. C., 1949.

* Another counter-example found after that of the referee is the following: $f(x)=$ $x \phi(x), x \neq 0, f(0)=0$, where $\phi(x)=1$ except in the intervals $\left[\left(1 / n-1 / n^{2}\right), 1 / n\right\}$, within which $\phi(x)=0$. Now $f(x)$ is continuous at $x=0$ and has no derivative there. But $1 / h \int_{0}^{h} \phi(x) d x \rightarrow 1$ as $h \rightarrow 0$, because the "dipped-out" area becomes an infinitesimal fraction of the whole (also infinitesimal) area between 0 and $h$, since as $h \sim 1 / n$, we remove $\sum_{\operatorname{man}}^{\infty} 1 / m^{3} \sim 1 / 2 n^{2} \sim 0(h)$. 
TABLE of $W(x) \equiv \sum_{n=1}^{\infty} \cos \left(7^{n} \pi x\right) / 2^{n}$

\begin{tabular}{|c|c|c|c|c|c|}
\hline$x$ & $\boldsymbol{H}^{\prime}(x)$ & & $x$ & $W(x)$ & \\
\hline .000 & 1.000000000000 & 1.000 & .050 & $.23088 \quad 91433 \quad 53$ & .950 \\
\hline .001 & .803915829849 & .999 & .051 & $.2068252628 \quad 39$ & .949 \\
\hline .002 & $.61188 \quad 60438 \quad 58$ & .998 & .052 & .271287157031 & .948 \\
\hline .003 & .537776037527 & .997 & .053 & $.1611834941 \quad 71$ & .947 \\
\hline .004 & $.64747 \quad 48039 \quad 38$ & .996 & .054 & .080695676970 & .946 \\
\hline .005 & $.87163 \quad 69853323$ & .995 & .055 & -.020661299004 & .945 \\
\hline .006 & $.76687 \quad 71957 \quad 75$ & .994 & .056 & -.114505519373 & .944 \\
\hline .007 & .608073455261 & .993 & .057 & -.022956525719 & .943 \\
\hline .008 & .435029407578 & .992 & .058 & -.019517246455 & .942 \\
\hline .009 & $.405+106+9+76$ & .991 & .059 & $.0115168818 \quad 80$ & .941 \\
\hline .010 & $.56641 \quad 31472 \quad 93$ & .990 & .060 & -.096981495280 & .940 \\
\hline .011 & .542753672027 & .989 & .061 & -.271873547272 & .939 \\
\hline .012 & .506949121598 & .988 & .062 & -.236530006345 & .938 \\
\hline .013 & .348012524587 & .987 & .063 & -.169656324421 & .937 \\
\hline .014 & $.22473 \quad 91530 \quad 39$ & .986 & .064 & $.01498 \quad 4363487$ & .936 \\
\hline .015 & .271964502668 & .985 & .065 & -.002397088580 & .935 \\
\hline .016 & $.2566587904 \quad 18$ & .984 & .066 & -.201819523674 & .934 \\
\hline .017 & $.345004843+78$ & $.98: 3$ & .067 & -.258563139523 & .933 \\
\hline .018 & .297440974020 & .982 & .068 & -.219325781704 & .932 \\
\hline .019 & .198960284226 & .981 & .069 & .055503381580 & .931 \\
\hline .020 & .162325470301 & .980 & .070 & .156909532647 & .930 \\
\hline .021 & .077727533597 & .979 & .071 & .014368399220 & .929 \\
\hline .022 & $.20584+479534$ & .978 & .072 & -.098120330488 & .928 \\
\hline .023 & .283635679636 & .977 & .073 & -.150745666850 & .927 \\
\hline .024 & $.31741 \quad 4736560$ & .976 & .074 & .092408849935 & .926 \\
\hline $.02 j$ & $.28730 \quad 1603897$ & .975 & .075 & .248905834007 & .925 \\
\hline .026 & .110542934142 & .974 & .076 & .206325925700 & .924 \\
\hline .027 & .172799430792 & .973 & .077 & .114623388235 & .923 \\
\hline .028 & $.2988159987 \quad 33$ & .972 & .078 & -.023041891960 & .922 \\
\hline .029 & .483728461058 & .971 & .079 & $.0955781653 \quad 49$ & .921 \\
\hline .030 & .544412194509 & .970 & .080 & .197940177319 & .920 \\
\hline .031 & .323889912278 & .969 & .081 & $\begin{array}{lll}.22531 & 98834 & 20\end{array}$ & .919 \\
\hline .032 & $.2699013283 \quad 84$ & .968 & .082 & .208765917694 & .918 \\
\hline .033 & .332257446204 & .967 & .083 & .053975775743 & .917 \\
\hline $.03 t$ & $\begin{array}{lll}58370 & 92580 & 29\end{array}$ & .966 & .084 & .048516604363 & .916 \\
\hline .035 & .749313015191 & .965 & .085 & $.02128 \quad 1974278$ & .915 \\
\hline .036 & .566320961185 & .964 & .086 & $.0368458507 \quad 18$ & .914 \\
\hline .037 & .433618648658 & .963 & .087 & $.08433 \quad 3768249$ & .913 \\
\hline .038 & .364962638350 & .962 & .088 & -.012143373142 & .912 \\
\hline .039 & .554359810640 & .961 & .089 & -.052152117101 & .911 \\
\hline .040 & $.75565 \quad 42269 \quad 37$ & .960 & .090 & -.195762284441 & .910 \\
\hline .041 & .661416118270 & .959 & .091 & -.263387422681 & .909 \\
\hline .042 & .542446760480 & .958 & .092 & -.218936017836 & .908 \\
\hline .043 & .365 .ั3 5306506 & .957 & .093 & -.229315008958 & .907 \\
\hline .044 & .411759059774 & .956 & .094 & -.192895454334 & .906 \\
\hline .040 & .545024682996 & .955 & .095 & -.360489445976 & .905 \\
\hline .046 & $.521786010 \lesssim 03$ & .954 & .096 & -.516246830442 & .904 \\
\hline .047 & $.49248 \quad 8867602$ & .953 & .097 & -.543500921487 & .903 \\
\hline .048 & .300886443750 & .952 & .098 & -.503501035440 & .902 \\
\hline \multirow[t]{2}{*}{.049} & $.22797 \quad 19914 \quad 12$ & .951 & .099 & -.338480678676 & .901 \\
\hline & $-W(x)$ & $x$ & & & $\boldsymbol{x}$ \\
\hline
\end{tabular}


TABLE OF A WEIERSTRASS CONTINUOUS NON-DIFFERENTIABLE FUNCTION

TABLE of $W(x)$-Continued

\begin{tabular}{|c|c|c|c|c|c|}
\hline$x$ & $W^{\prime}(x)$ & & $x$ & $W(x)$ & \\
\hline .100 & -.425325404176 & .900 & .150 & $-.60928 \quad 87419 \quad 74$ & .850 \\
\hline .101 & -.601220772899 & .899 & .151 & -.480526549966 & .849 \\
\hline .102 & -.714360466423 & .898 & .152 & -.497419707936 & .848 \\
\hline .103 & -.690322659569 & .897 & .153 & -.494797514565 & .847 \\
\hline .104 & $-.43794 \quad 7306453$ & .896 & .154 & $-.473 \tilde{3} 423 \tilde{2} 2265$ & $.8+6$ \\
\hline .105 & $-.40215 \quad 6353491$ & .895 & .155 & -.492919696368 & .845 \\
\hline .106 & -.506713093748 & .894 & .156 & -.36979 2885̄5 91 & .844 \\
\hline .107 & $-.65237 \quad 1746167$ & .893 & .157 & -.306770974135 & .843 \\
\hline .108 & -.687414647551 & .892 & .158 & $-.2191749907 \quad 16$ & .842 \\
\hline .109 & $-.44815 \quad 1239309$ & .891 & .159 & -.215 .316998341 & .841 \\
\hline .110 & -.339489049228 & .890 & .160 & $-.3277+87639 \quad 11$ & .840 \\
\hline .111 & -.326968369691 & .889 & .161 & $-.30751 \quad 7525062$ & $.8: 39$ \\
\hline .112 & $-.42541 \quad 62768 \quad 18$ & .888 & .162 & -.270205665927 & $.8: 38$ \\
\hline .113 & -.507012502615 & .887 & .163 & -.114947817484 & .837 \\
\hline .114 & -.365270405346 & .886 & .164 & -.056937644149 & $.8: 36$ \\
\hline .115 & $-.2871702983 \quad 14$ & .885 & .165 & -.195422966860 & $.8: 35$ \\
\hline .116 & -.194358653972 & .884 & .166 & -.301528394441 & .834 \\
\hline .117 & -.203430154989 & .883 & .167 & -.383663917931 & .833 \\
\hline .118 & -.276489528796 & .882 & .168 & -.229093944073 & .8 .32 \\
\hline .119 & -.240918706161 & .881 & .169 & -.095009141695 & $.8: 31$ \\
\hline .120 & -.274278958066 & .880 & .170 & -.173038009899 & .830 \\
\hline .121 & -.195945370568 & .879 & .171 & -.338066797386 & .829 \\
\hline .122 & $-.14745 \quad 62719 \quad 19$ & .878 & .172 & -.550640283216 & .828 \\
\hline .123 & -.160779076002 & .877 & .173 & -.465847811099 & .827 \\
\hline .124 & -.163131171657 & .876 & .174 & -.301298374107 & .826 \\
\hline 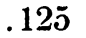 & -.307959844170 & .875 & .175 & -.278036956560 & .825 \\
\hline .126 & -.327790791831 & .874 & .176 & -.392806593650 & .824 \\
\hline .127 & -.306421790698 & .873 & .177 & -.651827985226 & $.82: 3$ \\
\hline .128 & -.254575499271 & .872 & .178 & -.651823938820 & .822 \\
\hline .129 & -.205212864540 & .871 & .179 & -.533510237189 & .821 \\
\hline .130 & -.382265700618 & .870 & .180 & -.442684427472 & .820 \\
\hline .131 & -.510086053512 & .869 & .181 & -.435780102183 & .819 \\
\hline .132 & -.588978829307 & .868 & .182 & $-.6198587140 \quad 15$ & .818 \\
\hline .133 & -.516050321822 & .867 & .183 & -.649222169263 & .817 \\
\hline .134 & -.372280255549 & .866 & .184 & -.622778743301 & .816 \\
\hline .135 & -.482224013576 & .865 & .185 & -.544416543173 & $.81 j$ \\
\hline .136 & -.644769094244 & .864 & .186 & -.434165110185 & .814 \\
\hline .137 & -.826566089190 & .863 & .187 & -.471534372297 & .813 \\
\hline .138 & -.789005024213 & .862 & .188 & -.441878591252 & .812 \\
\hline 139 & -.584602058218 & .861 & .189 & -.483243465321 & .811 \\
\hline .140 & -.580176101865 & .860 & .190 & -.481004554476 & .810 \\
\hline .141 & -.673589329465 & .859 & .191 & -.362102463976 & .809 \\
\hline .142 & -.883349774078 & .858 & .192 & -.283850725058 & .808 \\
\hline .143 & -.901955447525 & .857 & .19 .3 & -.139573437896 & .807 \\
\hline .144 & -.717356798431 & .856 & .194 & -.171327351109 & .806 \\
\hline .145 & $-.6354271888 \quad 15$ & .855 & .195 & -.243809120797 & .805 \\
\hline .146 & -.601728492947 & .854 & .196 & -.218707253252 & .804 \\
\hline .147 & -.739790581132 & .853 & .197 & -.138387132299 & .803 \\
\hline .148 & -.779966135853 & .852 & .198 & .098018236071 & .802 \\
\hline \multirow[t]{2}{*}{.149} & -.678212362364 & .851 & .199 & .146665232761 & .801 \\
\hline & $-W^{\prime}(x)$ & $x$ & & $-W(x)$ & $x$ \\
\hline
\end{tabular}


TABLE of $W(x)$-Continued

\begin{tabular}{|c|c|c|c|c|c|}
\hline$x$ & $\mathbb{N}^{\prime}(x)$ & & $x$ & $\Psi^{\prime}(x)$ & \\
\hline .200 & $.063366 \quad 10018 \quad 75$ & .800 & .250 & .707106781187 & .750 \\
\hline .201 & $-.041752236+04$ & .799 & .251 & $\begin{array}{lll}.59986 & 16383 & 91\end{array}$ & .749 \\
\hline .202 & $-.07+76 \quad 70750 \quad 18$ & .798 & .252 & $.42999 \quad 19525 \quad 71$ & .748 \\
\hline $.20: 3$ & $16401 \quad 5522004$ & .797 & .2533 & .366609323594 & .747 \\
\hline .204 & .99971 35815 61 & .796 & .254 & .327956254406 & $.7+6$ \\
\hline .205 & $.28: 36 \quad 84: 37500$ & .795 & .255 & $.45218 \quad 1113491$ & .745 \\
\hline .206 & $.10033 \quad 1267+93$ & .794 & .256 & .430007860724 & .744 \\
\hline .207 & $-.08006 .3650+29$ & $.79: 3$ & $.25 \pi$ & .382707093421 & .743 \\
\hline .208 & $.0660253: 22800$ & .792 & .258 & $.32431 \quad 2379108$ & $.7+2$ \\
\hline .209 & $.21948: 390979$ & .791 & .259 & $.18040 \quad 34507 \quad 72$ & $.7+1$ \\
\hline .210 & $.99866 \quad 9: 3766$ t3 & .790 & .260 & $.20082 \quad 17490 \quad 15$ & .740 \\
\hline .211 & $.14: 3+70010207$ & .789 & .261 & .195029228286 & .739 \\
\hline .212 & $\begin{array}{lll}-.11156 & 27605 & 97\end{array}$ & .788 & .262 & $.28277 \quad 1153362$ & $.7: 38$ \\
\hline .213 & $-.118927610+733$ & .787 & .263 & $.33124941+3 \quad 10$ & .737 \\
\hline .214 & $-.00503 \quad 4.368: 39.3$ & .786 & .264 & .195358370439 & .736 \\
\hline .215 & $.12393 \quad 18196+4$ & $.78 i$ & .265 & .131300193473 & $.73 j$ \\
\hline .216 & 072370252068 & .784 & .266 & .059239700164 & .734 \\
\hline .217 & -.128706688132 & $.78: 3$ & .267 & $.2032081841 \quad 97$ & .7333 \\
\hline .218 & -1719063311985 & .782 & .268 & .380973017589 & .732 \\
\hline .219 & $-.205339+622+39$ & .781 & .269 & $\begin{array}{lll}.36488 & 72388 & 22\end{array}$ & .731 \\
\hline .220 & -.10396 9335i)1 29 & .780 & .270 & $\begin{array}{lll}.30069 & 58598 & 63\end{array}$ & .730 \\
\hline .21 & - 0.3670 84900 j3 & .779 & .271 & .128889622058 & .729 \\
\hline .222 & $-.110580+97998$ & .778 & .272 & .219308857152 & .728 \\
\hline .223 & $-11053+7420+1$ & .775 & $.27: 3$ & $.45450 \quad 66523 \quad 44$ & .727 \\
\hline .224 & $-2 \cdot 2 \cdot 263: 98542+4$ & .776 & .274 & $.58788 \quad 37027 \quad 28$ & .726 \\
\hline .225 & $-.2(0+332052+80$ & .775 & .275 & $.61063378772 \quad 02$ & .725 \\
\hline .226 & $-.13899 \quad 1+77388$ & .774 & .276 & $.37897 \quad 1170932$ & .724 \\
\hline .227 & $-11533385227+30$ & $.77: 3$ & .277 & $.3549100906 \quad 37$ & .723 \\
\hline .228 & $.1717991975+4$ & .772 & .278 & $.53331701360 \quad 59$ & .722 \\
\hline $2 \cdot 2 !$ & $-.01896 \quad 6289875$ & .771 & .279 & $.74080 \quad 38401 \quad 87$ & .721 \\
\hline $.2: 30$ & -071132971174 & .770 & .280 & $.873884+6+1 \quad 26$ & .720 \\
\hline .231 & $-.183351+906+96$ & .769 & .281 & $.66344+129097$ & .719 \\
\hline .232 & $.19: 311672(0+1875$ & .768 & .282 & $.55360 \quad 4431088$ & .718 \\
\hline $.2: 3: 3$ & $28+702: 34+534$ & .767 & .283 & $.598 .78 \quad 967+752$ & .717 \\
\hline .234 & $\therefore(): 37306642148$ & .766 & .284 & $\begin{array}{llll}.75311 & 92971 & 19\end{array}$ & .716 \\
\hline .2:35 & $25.3: 3156944685$ & .765 & $.28 \%$ & $.9357 \% 6808902$ & .715 \\
\hline $.2: 36$ & 12240787784 & .764 & .286 & $.80593 \quad 3152: 3 \quad 57$ & .714 \\
\hline .237 & $106091+6178 \quad 36$ & .763 & .287 & .70250 .5478563 & .713 \\
\hline .238 & $4.3+16: 31698 \quad 35$ & .162 & .288 & $.62769 \quad 787.35 \quad 12$ & .712 \\
\hline .239 & הi2.74 (0)3757 22 & .761 & .289 & .640513152434 & .711 \\
\hline .240 & (ifor):20 $7+(0: 7793$ & .760 & .290 & $.7699870795 \quad 56$ & .710 \\
\hline .241 & .:(1)!1:3 37880 48 & .759 & .291 & $.71343 \quad 52538 \quad 50$ & .709 \\
\hline .242 & $29(4912109120$ & .758 & .292 & .701110242736 & .708 \\
\hline .243 & $.77 ; 233 \quad 8(i 33: 394 \quad 28$ & $.7 \% 7$ & .293 & .59700 $86384 \quad 17$ & .707 \\
\hline .244 &.$(6.5+52 \quad(02702 \quad 15$ & .756 & .294 & $.48196 \quad 39792 \quad 70$ & .706 \\
\hline .245 & $780+9973: 32619$ & .755 & .295 & $.48841 \quad 28610 \quad 21$ & .705 \\
\hline .246 & .587-1 1344618 & .754 & .296 & $.43820: 3271149$ & .704 \\
\hline .247 & 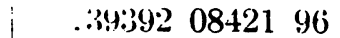 & .753 & .297 & .032468913876 & .703 \\
\hline .248 & $43533+5589280$ & .752 & .298 & $.50028+764504$ & .702 \\
\hline .249 & .537047031188 & .7 .51 & .299 & .364153318500 & .701 \\
\hline & - & $x$ & & & $x$ \\
\hline
\end{tabular}


TABLE of $W(x)$-Continued

\begin{tabular}{|c|c|c|c|c|c|}
\hline$x$ & $W(x)$ & & $x$ & $W^{\prime}(x)$ & \\
\hline .300 & .262865556060 & .700 & .350 & .007787786967 & .650 \\
\hline .301 & .145829858987 & .699 & .351 & -.172042208650 & .649 \\
\hline .302 & $.2856: 39740701$ & 698 & .352 & -.230292124186 & .648 \\
\hline .303 & .366335015787 & .697 & .353 & -.236473086444 & .647 \\
\hline .304 & $.3328281740 \quad 47$ & .696 & .354 & $-.01762 \quad 2527442$ & .646 \\
\hline .305 & 21458 96315.30 & .695 & .355 & $.0666922195 \quad 13$ & 645 \\
\hline .306 & $.00939 \quad 20926 \quad 4 j$ & .694 & .356 & .000756269021 & $.6+4$ \\
\hline .307 & .107108007880 & .693 & .357 & -.0206 .37523612 & .643 \\
\hline .308 & $.2562+9170422$ & .692 & .358 & $-.08880 \quad 13434 \quad 64$ & $.6+2$ \\
\hline .309 & .378 .389794527 & .691 & .359 & .039807768840 & $.6+1$ \\
\hline .310 & .343852008552 & .690 & .360 & .100066084169 & .640 \\
\hline .311 & $.0987 \check{5} 62075 \quad 19$ & .689 & .361 & $.12219895 \overline{3} 34$ & .639 \\
\hline .312 & .107374648370 & .688 & .362 & .187375555883 & .638 \\
\hline $.31: 3$ & $.23160 \quad 40973 \quad 59$ & .687 & .363 & .109542131700 & .637 \\
\hline .314 & .455356200252 & .686 & .364 & .128198733070 & .636 \\
\hline .315 & $.54102 \quad 65 \check{479} 93$ & .685 & .365 & $.07610 \quad 3882989$ & .635 \\
\hline .316 & .337362835770 & .684 & .366 & $.09741 \quad 5611137$ & .634 \\
\hline .317 & .283558670620 & .683 & .367 & .228356637641 & .633 \\
\hline .318 & .309685218951 & .682 & .368 & .197963587829 & .632 \\
\hline .319 & $.51427 \quad 3737170$ & .681 & .369 & $.16937 \quad 55518 \quad 30$ & .631 \\
\hline .320 & .664588016607 & .680 & .370 & $-.0056233217 \quad 53$ & .630 \\
\hline .321 & .553830222236 & .679 & .371 & -.071895736001 & .629 \\
\hline .322 & .513060836700 & .678 & .372 & .046040839666 & .628 \\
\hline .323 & $.4386+1418698$ & .677 & .373 & $.07904 \quad 58408 \quad 70$ & .627 \\
\hline .324 & $\begin{array}{lll}.52349 & 24425 \quad 12\end{array}$ & .676 & .374 & $.097088476+28$ & .626 \\
\hline .325 & $.6300+2962766$ & .67う & .375 & $-.127 j 6 \quad 1144122$ & .625 \\
\hline .326 & .593087296 .501 & .674 & .376 & $-.30043 \quad 5011772$ & $.62 t$ \\
\hline .327 & $.6279+49761307$ & .673 & .377 & $-.27256 \quad 67762 \quad 10$ & $.62: 3$ \\
\hline .328 & $.5180 \Xi 4127103$ & .672 & .378 & -.212567851798 & .622 \\
\hline .329 & $.47323 \quad 47330 \quad 89$ & .671 & .379 & $-.0942694456 \quad 39$ & .621 \\
\hline .330 & .452967693202 & .670 & .380 & -.2666326380178 & .620 \\
\hline .331 & .413659576699 & .669 & .381 & $-.4804 \check{5} 1908693$ & .619 \\
\hline .332 & $.52433 \quad 3536236$ & .668 & .382 & -.556452319561 & .618 \\
\hline .333 & .459997192026 & .667 & $.38: 3$ & -.526375208999 & .617 \\
\hline .334 & .370141234331 & .666 & .384 & -.33321250132429 & .616 \\
\hline .335 & .225520223519 & .665 & .385 & $-.3947974+1787$ & . $61 j$ \\
\hline .336 & $.109045 \check{5} 45952$ & .664 & .386 & -.546570792333 & .614 \\
\hline .337 & .234213776308 & .6633 & .387 & -.661928470916 & .613 \\
\hline .338 & .253032342937 & .662 & .388 & -.690405329205 & .612 \\
\hline .339 & $.23376 \quad 3293751$ & .661 & .389 & $-.49978 \quad 3789632$ & .611 \\
\hline .340 & .050899086254 & .660 & .390 & -.481003862593 & .610 \\
\hline .341 & -.157169066972 & .659 & .391 & -.504447925890 & .609 \\
\hline .342 & -.090447294999 & .658 & .392 & -..5618:3 0583268 & .608 \\
\hline .343 & -.017529421589 & .657 & .393 & -.623185880380 & .607 \\
\hline .344 & .096997517951 & .656 & .394 & -.507782619653 & .606 \\
\hline .345 & -.016889367223 & .655 & .395 & -.496473394832 & .605 \\
\hline .346 & $-.2565+0916879$ & .654 & .396 & $-.4130252891+0$ & $.60 t$ \\
\hline .347 & -.273828859764 & $.65: 3$ & .397 & $-.35 \overline{5} 899213583$ & .603 \\
\hline .348 & -.214638285053 & .652 & .398 & -.388678221092 & .602 \\
\hline \multirow[t]{2}{*}{.349} & .001842583 .582 & .651 & .399 & -.357916348126 & .601 \\
\hline & & $x$ & & & 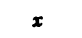 \\
\hline
\end{tabular}


TABLE of $W(x)$-Concluded

\begin{tabular}{|c|c|c|c|c|c|}
\hline$x$ & $H(x)$ & & $x$ & $H^{\prime}(x)$ & \\
\hline .400 & -.436338998125 & .600 & .450 & -.140858891107 & .550 \\
\hline .401 & -.341086485367 & .599 & .451 & -.287018570394 & .549 \\
\hline .402 & $-.1999 j 66617 \quad 14$ & .598 & .452 & -.406623055260 & .548 \\
\hline .403 & $-.156248+34239$ & .597 & .453 & -.260535891944 & .547 \\
\hline .404 & -.153443186489 & .596 & .454 & -.097926998633 & $.5+6$ \\
\hline .405 & $-.33660+273789$ & .595 & .455 & -.145695409097 & .545 \\
\hline .406 & -.330070659737 & .594 & .456 & -.269840936285 & .544 \\
\hline .407 & -.204524663206 & .593 & .457 & -.478762406797 & .543 \\
\hline.+08 & $-.09102+226568$ & .592 & .458 & -.449072853516 & .542 \\
\hline .409 & -.041116796583 & .591 & .459 & -.347533693600 & .541 \\
\hline .410 & $-.26774+462533$ & .590 & .460 & -.33327 1943823 & .540 \\
\hline.+11 & -.382743337509 & .589 & .461 & -.351462576411 & .539 \\
\hline .412 & $-.36998 \quad \jmath 144242$ & .588 & .462 & $-.52273 \quad 6207443$ & .538 \\
\hline .413 & -.2468945330700 & .587 & .463 & -.559874126767 & .537 \\
\hline .414 & -.117367839284 & .586 & .464 & -.573440633245 & .536 \\
\hline .415 & $-.29765 \quad 178+4 \quad 11$ & .585 & .465 & -.574046955042 & .535 \\
\hline .416 & -.474942149222 & .584 & .466 & -.481362266042 & .534 \\
\hline.+17 & -.596558491874 & .583 & .467 & -.514276190127 & .533 \\
\hline.+18 & $-.53275 \quad 3680459$ & .582 & .468 & -.513013566251 & .532 \\
\hline .419 & -.359683100154 & .581 & .469 & -.614361772969 & .531 \\
\hline .420 & -.442652877724 & .580 & .470 & -.691447066605 & .530 \\
\hline .421 & -.573676815200 & .579 & .471 & -.563926376792 & .529 \\
\hline .422 & $-.75568 \quad 10645 \quad 53$ & .578 & .472 & -.455200438735 & .528 \\
\hline .423 & -.773431680693 & .577 & .473 & -.329119232810 & .527 \\
\hline .424 & -.632426172682 & .576 & .474 & -.425403235539 & .526 \\
\hline .425 & -.642109468815 & .575 & .475 & -.576270763741 & .525 \\
\hline.+26 & -.647921262064 & .574 & .476 & -.513978368936 & .524 \\
\hline .427 & -.771078310191 & .573 & .477 & -.359133792298 & .523 \\
\hline .428 & -.823697924071 & .572 & .478 & -.104305080599 & .522 \\
\hline .429 & -.768865812318 & .571 & .479 & -.104549917966 & .521 \\
\hline .430 & -.782959853829 & .570 & .480 & -.262922687867 & .520 \\
\hline .431 & -.671789512299 & .569 & .481 & -.317068197654 & .519 \\
\hline .432 & $-.6572868184 \quad 10$ & .568 & .482 & -.241336234353 & .518 \\
\hline .433 & -.659575657242 & .567 & .483 & .057625073401 & .517 \\
\hline .434 & -.678922621460 & .566 & .484 & .172881203015 & .516 \\
\hline $.43 \overline{3}$ & -.767524170356 & .565 & .485 & .086277988317 & .515 \\
\hline .436 & -.628986550620 & .564 & .486 & -.051539303959 & .514 \\
\hline .437 & -.497193980006 & .563 & .487 & -.120444889659 & .513 \\
\hline .438 & -.388380690326 & .562 & .488 & .107050321466 & .512 \\
\hline .439 & -.411535915314 & .561 & .489 & .266940692326 & .511 \\
\hline .440 & -.583261669224 & .560 & .490 & $.2824083062 \quad 16$ & .510 \\
\hline .441 & -.52360 7097404 & .559 & .491 & .150281642695 & .509 \\
\hline .442 & -.381908298612 & .558 & .492 & -.023617557402 & .508 \\
\hline .443 & -.174430123101 & .557 & .493 & .066843893329 & .507 \\
\hline .444 & -.127787944171 & .556 & .494 & $.1587 \tilde{5} 42472 \quad 12$ & .506 \\
\hline .445 & -.324136866258 & .555 & .495 & .232156321975 & .505 \\
\hline .446 & -.389994440708 & .554 & .496 & .183674621092 & .504 \\
\hline 447 & -.35689245335 27 & .553 & .497 & .019312160007 & .50 .3 \\
\hline .448 & -.132668438220 & .552 & 498 & .003785592821 & .502 \\
\hline \multirow[t]{3}{*}{.449} & .000590698460 & .551 & .499 & $-.0444166347 \quad 11$ & .501 \\
\hline & & & .500 & .000000000000 & .500 \\
\hline & $-u$ & $x$ & & $-W^{\prime}(x)$ & 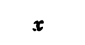 \\
\hline
\end{tabular}

